\title{
Parallel re-modeling of EF-1 a function: divergent EF-1a genes co-occur with EFL genes in diverse distantly related eukaryotes
}

\author{
Ryoma Kamikawa ${ }^{1,2^{*}}$, Matthew W Brown ${ }^{3}$, Yuki Nishimura ${ }^{4}$, Yoshihiko Sako ${ }^{5}$, Aaron A Heiss ${ }^{6}$, Naoji Yubuki ${ }^{7}$ \\ Ryan Gawryluk ${ }^{3}$, Alastair GB Simpson ${ }^{6}$, Andrew J Roger ${ }^{3}$, Tetsuo Hashimoto ${ }^{4,8}$ and Yuji Inagaki ${ }^{4,8}$
}

\begin{abstract}
Background: Elongation factor-1a (EF-1a) and elongation factor-like (EFL) proteins are functionally homologous to one another, and are core components of the eukaryotic translation machinery. The patchy distribution of the two elongation factor types across global eukaryotic phylogeny is suggestive of a 'differential loss' hypothesis that assumes that EF-1 $a$ and EFL were present in the most recent common ancestor of eukaryotes followed by independent differential losses of one of the two factors in the descendant lineages. To date, however, just one diatom and one fungus have been found to have both EF-1a and EFL (dual-EF-containing species).

Results: In this study, we characterized 35 new EF-1a/EFL sequences from phylogenetically diverse eukaryotes. In so doing we identified 11 previously unreported dual-EF-containing species from diverse eukaryote groups including the Stramenopiles, Apusomonadida, Goniomonadida, and Fungi. Phylogenetic analyses suggested vertical inheritance of both genes in each of the dual-EF lineages. In the dual-EF-containing species we identified, the EF-1a genes appeared to be highly divergent in sequence and suppressed at the transcriptional level compared to the co-occurring EFL genes.

Conclusions: According to the known EF-1a/EFL distribution, the differential loss process should have occurred independently in diverse eukaryotic lineages, and more dual-EF-containing species remain unidentified. We predict that dual-EF-containing species retain the divergent EF-1a homologues only for a sub-set of the original functions. As the dual-EF-containing species are distantly related to each other, we propose that independent re-modelling of EF-1 a function took place in multiple branches in the tree of eukaryotes.
\end{abstract}

Keywords: Diatoms, Differential Gene Loss, EF-1a, EFL, Functional Remodeling, Goniomonas, Pythium, Spizellomyces, Thecamonas

\section{Background}

Elongation factor $1 \alpha(\mathrm{EF}-1 \alpha)$ proteins in eukaryotes and archaebacteria, and their orthologues in bacteria (elongation factor $\mathrm{Tu}$ ), are GTPases required for the central process of translation $[1,2]$. The primary sequence of EF$1 \alpha$ is highly conserved across the tree of life, suggesting that this protein was established in the last universal

\footnotetext{
* Correspondence: kamikawa.ryoma.7v@kyoto-u.ac.jp

'Graduate School of Global Environmental Studies, Kyoto University, Kyoto 606-8501, Japan

${ }^{2}$ Graduate School of Human and Environmental Studies, Kyoto University, Kyoto 606-8501, Japan

Full list of author information is available at the end of the article
}

common ancestor, and inherited by extant organisms [3]. However, genomic and transcriptomic data from diverse organisms have shown that some eukaryotic lineages lack EF-1 $\alpha$, and these lineages instead were found to possess a putative EF-1 $\alpha$-related GTPase [4]. These elongation factor-like (EFL) proteins are believed to perform the same function in translation as EF-1 $\alpha$, as there is no significant functional divergence in the regions that are critical for EF-1 $\alpha$ function [4]. The functional equivalence of EFL and EF- $1 \alpha$ would explain the mutually exclusive distributions of EFL and EF- $1 \alpha$ genes amongst eukaryotes since EF- $1 \alpha$ would be functionally

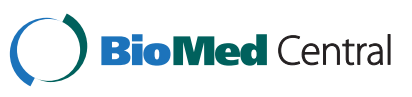


redundant in eukaryotes with EFL-mediated translation elongation, and vice versa.

Intensive surveys for EFL genes in phylogenetically diverse eukaryotes revealed a number of groups that have both 'EF-1 $\alpha$-containing' and 'EFL-containing' species [5-10]. The co-existence of EF-1 $\alpha$-containing and EFL-containing species in a monophyletic group can be explained by the ancestral co-occurrence of EF$1 \alpha$ and EFL, and subsequent losses of either of the two elongation factors in the descendants. Henceforth, we designate the above scenario simply as the 'differential loss' hypothesis [8]. Many aspects of this hypothesis are difficult to test experimentally. Nonetheless, dual expression of EF- $1 \alpha$ and EFL proteins in Trypanosoma brucei cells, which corresponds to the ancestral state assumed in the differential loss hypothesis, had no apparent impact on cell viability [11].

It was previously found that examined diatom species were either EF-1 $\alpha$-containing or EFL-containing, except for a single species, Thalassiosira pseudonana, whose genome encodes both EF-1 $\alpha$ and EFL genes [7]. According to the differential loss hypothesis described above, the EF-1 $\alpha / E F L$ gene data from diatoms can be explained as follows: (1) the ancestral diatom genome was 'dual-EF-containing,' (2) the T. pseudonana genome retains the ancestral state, and (3) the EF- $1 \alpha$ (or EFL) gene was lost in the extant EFL-containing (or EF-1 $\alpha$ containing) descendants [7]. A similar situation has been proposed for Fungi; although the vast majority of fungal species are either EF-1 $\alpha$-containing or EFL-containing, a single species, Basidiobolus ranarum, was found to be dual-EF-containing [12]. Under the differential loss hypothesis, $T$. pseudonana and B. ranarum retain the ancestral state of diatom and fungal genomes, respectively.

The differential loss hypothesis is an increasingly popular explanation of the current EF- $1 \alpha / E F L$ gene distribution in the tree of eukaryotes. Nevertheless, dual-EF-containing species, which are believed to reflect the ancestral state of their phylogenetic relatives containing either EF- $1 \alpha$ or EFL, have, to date, only been described in diatoms and Fungi. In this study, by experimental surveys and data mining in publicly available genome and/or transcriptomic data, four independent lineages-Stramenopiles, Apusomonadida, Goniomonadida, and Fungi-were found to contain at least one dual EF-containing species (11 species were newly identified in total). All EF- $1 \alpha$ genes in the dual EF-containing species examined here appear to be divergent, and are transcribed at a much lower level than the co-occurring EFL genes, suggesting that EF- $1 \alpha$ has functionally diverged in these species. We propose that the re-modeling of the original EF- $1 \alpha$ functions seemingly occurred in several independent branches of the tree of eukaryotes.

\section{Results}

We successfully isolated/identified 20 and 15 previously unidentified EF- $1 \alpha$ and EFL sequences, respectively, by a PCR survey or mining publicly available and in-house genomic/transcriptomic databases (Table 1). Five diatoms, three oomycetes, one goniomonad, one apusomonad, and a chytridiomycete fungus were found to be dual-EF-containing in this study, in addition to the two previously reported dual-EF-containing species, the diatom T. pseudonana [7] and a fungus of uncertain taxonomic affiliation, $B$. ranarum [12]. We updated EF- $1 \alpha$ and EFL alignments by adding the new sequences listed in Table 1, and both alignments were analyzed with maximum-likelihood (ML) and Bayesian phylogenetic methods (Figures 1 and 2).

\section{Dual-EF-containing species in diatoms}

The majority of diatom species, in which EF- $1 \alpha / E F L$ sequences have been characterized to date, appear to possess EFL genes, except for the genomes of Phaeodactylum tricornutum [13], which encodes only an EF-1 $\alpha$ gene, and T. pseudonana, which encodes both EF- $1 \alpha$ and EFL genes [7]. In this study, we surveyed EF-1 $\alpha / E F L$ genes in diatoms further, and identified five more dual-EFcontaining species, indicating that dual-EF-containing species are quite prevalent amongst diatoms. EF-1 $\alpha$ transcripts were detected in Detonula confervacea, Achnanthes kuwaitensis, Fragilariopsis cylindrus, Thalassionema nitzschioides, and Asterionella glacialis, all of which were previously considered to be 'EFL-containing'. In the EF-1 $\alpha$ ML tree, all diatom homologues grouped together with an ML bootstrap value (MLBP) of 57\% (node A in Figure 1), and this group branches with the EF- $1 \alpha$ homologues of the bolidophyte Bolidomonas pacifica. Although the statistical support for the diatom-Bolidomonas affiliation was moderate (MLBP $=75 \%$; node B in Figure 1 ), this particular affiliation found in the EF- $1 \alpha$ phylogeny is consistent with their close (organismal) relationships [14]. Thus we concluded that there had been vertical descent of EF- $1 \alpha$ genes in the diatom-Bolidomonas clade. As shown in previous studies e.g., [7], the updated EFL phylogeny also includes a diatom clade, indicating the vertical descent of EFL genes in this lineage (Figure 2).

Quantitative reverse transcriptase PCR (qRT-PCR) assays revealed that the expression level of the EFL gene is much greater than that of the EF- $1 \alpha$ gene in each of the dual EF-containing diatom species identified in this study (Table 2), except for F. cylindrus, for which these assays were not performed. However, EF-1 $\alpha$ transcripts are likely much less abundant than EFL transcripts in F. cylindrus as well, since only EFL transcripts were detected in the F. cylindrus transcriptomic data publicly available from the Joint Genome Institute (http://genome.jgi.doe.gov/). 
Table $1 \mathrm{EF}-1 \mathrm{a}$ and EFL homologues isolated/identified in this study

\begin{tabular}{|c|c|c|c|c|}
\hline Gene & Taxon name & Classification & Survey & Accession nos. \\
\hline$E F-1 a^{*}$ & Detonula confervcace CCMP353 & Diatoms & PCR & AB766031 \\
\hline$E F-1 a^{*}$ & Achnanthes kuwaitensis NIES1349 & Diatoms & PCR & AB775895 \\
\hline$E F-1 a^{*}$ & Fragilariopsis cylindrus & Diatoms & Genome, public & See Additional file 3 \\
\hline$E F-1 a^{*}$ & Thalassionema nitzschioides NIES534 & Diatoms & PCR & AB766032 \\
\hline$E F-1 a^{*}$ & Asterionella glacialis NIES417 & Diatoms & PCR & AB766030 \\
\hline EF-1a & Bolidomonas pacifica CCMP1866 & Bolidophyceae & PCR & AB766033 \\
\hline$E F-1 a^{*}$ & Pythium intermedium MAFF306022 & Oomycetes & $P C R$ & AB766039 \\
\hline \multirow[t]{2}{*}{$E F-1 a^{*}$} & Pythium ultimum DAOM BR144 & Oomycetes & Genome, public & See Additional file 3 \\
\hline & & & EST, public & \\
\hline$E F-1 a^{*}$ & Pythium apleroticum MAFF425515 & Oomycetes & $P C R$ & AB766038 \\
\hline EF-1a & Goniomonas sp. ATCC PRA68 & Goniomonadida & PCR & AB766034 \\
\hline$E F-1 a^{*}$ & Goniomonas sp. ATCC 50180 & Goniomonadida & PCR & AB766037 \\
\hline$E F-1 a$ & Goniomonas truncata NIES 1373 & Goniomonadida & PCR & AB766036 \\
\hline EF-1a & Goniomonas sp. CCAP 980_1 & Goniomonadida & $P C R$ & AB766035 \\
\hline \multirow[t]{2}{*}{$E F-1 a^{*}$} & Spizellomyces punctatus DAOM BR117 & Chytridiomycota & Genome, public & See Additional file 3 \\
\hline & & & EST, public & \\
\hline EF-1a & Subulatomonas sp. strain PCMinv5 & Breviata & EST, in-house & AB766043 \\
\hline$E F-1 a$ & Breviata-like biflagellate strain PCbi66 & Breviata & EST, in-house & AB766042 \\
\hline$E F-1 a$ & Roombia sp. NY0200 & Katablepharida & PCR & AB766040 \\
\hline EF-1a & Mantamonas plastica Bass1 (CCAP 1946/1) & Mantamonadida & EST, in-house & AB766041 \\
\hline \multirow[t]{2}{*}{$E F-1 a^{*}$} & Thecamonas trahens ATCC50062 & Apusomonadida & EST, public & See Additional file 3 \\
\hline & & & Genome, public & \\
\hline EF-1a & microaerophilic cercozoan strain DMV & Filosa & EST, in-house & AB824019 \\
\hline $\mathrm{EFL}^{\natural}$ & Goniomonas sp. ATCC 50180 & Goniomonadida & PCR & AB766045 \\
\hline EFL & Goniomonas sp. NIES 1374 & Goniomonadida & PCR & AB766044 \\
\hline EFL & Pythium spinosum MAFF425453 & Oomycetes & $P C R$ & AB766051 \\
\hline $\mathrm{EFL}^{\natural}$ & Pythium intermedium MAFF306022 & Oomycetes & PCR & AB766049 \\
\hline EFL & Pythium uncinulatum MAFF240295 & Oomycetes & PCR & AB766052 \\
\hline EFL & Pythium conidiophorum MAFF245320 & Oomycetes & $P C R$ & AB766047 \\
\hline EFL & Pythium porphyrae MAFF239483 & Oomycetes & PCR & AB766050 \\
\hline EFL" & Pythium apleroticum MAFF425515 & Oomycetes & $P C R$ & AB766046 \\
\hline EFL & Pythium echinulatum MAFF425510 & Oomycetes & $P C R$ & AB766048 \\
\hline $\mathrm{EFL}$ & Pythium insidiosum CBS119452 & Oomycetes & EST, public & See Additional file 3 \\
\hline \multirow[t]{2}{*}{$\mathrm{EFL}^{\natural}$} & Spizellomyces punctatus DAOM BR117 & Chytridiomycota & Genome, public & See Additional file 3 \\
\hline & & & EST, public & \\
\hline \multirow[t]{2}{*}{$\mathrm{EFL}^{\natural}$} & Thecamonas trahens ATCC50062 & Apusomonadida & Genome, public & See Additional file 3 \\
\hline & & & EST, public & \\
\hline EFL & Capromyxa protea CF08-5 (ATCC PRA-324) & Tubulinida & EST, in-house & AB766053 \\
\hline EFL & Fabomonas tropica strain NYK3C & Ancyromonadida & EST, in-house & AB766055 \\
\hline EFL & Ancyromonas sigmides strain B70 (CCAP 1958/3) & Ancyromonadida & EST, in-house & AB766054 \\
\hline
\end{tabular}

*co-occurred with EFL, "co-occurred with EF-1a. Accession numbers for the sequences obtained by public database search are not described, but their protein sequences were shown in Additional file 3. 


\section{Dual-EF-containing species in oomycetes}

Only EF-1 $\alpha$ homologues were identified in well-studied members of the Oomycetes (e.g., Phytophthora infestans, for which a complete genome is available [15]), but some of us have recently reported EFL genes in Pythium oligandrum and Pythium ultimum [16]. In this study, we resurveyed EF-1 $\alpha / E F L$ sequences in 8 members of the genus Pythium, and identified Pythium intermedium, Py. ultimum, and Py. apleroticum as dual-EF-containing. The EF-1 $\alpha$ phylogenetic analysis successfully recovered the monophyly of all of oomycetes, suggesting that Py. intermedium, Py. ultimum, and Py. apleroticum vertically inherited their EF-1 $\alpha$ genes from a common oomycete ancestor. We suspect that the ML bootstrap support for the oomycete clade in the EF- $1 \alpha$ analysis was lowered due to the divergent nature of the Py. intermedium, Py. ultimum, and Py. apleroticum homologues (MLBP $=22 \%$; node $C$ in Figure 1). The EFL phylogeny also robustly unites all oomycete EFL sequences, including those of the three dual-EF-containing Pythium spp. (Figure 2).

The EF- $1 \alpha$ gene of Py. ultimum is seemingly much less transcribed than its EFL gene. In Illumina transcriptomic data, the k-mer frequency for EFL contig was significantly higher than that for a cytoskeletal protein, $\alpha$-tubulin 


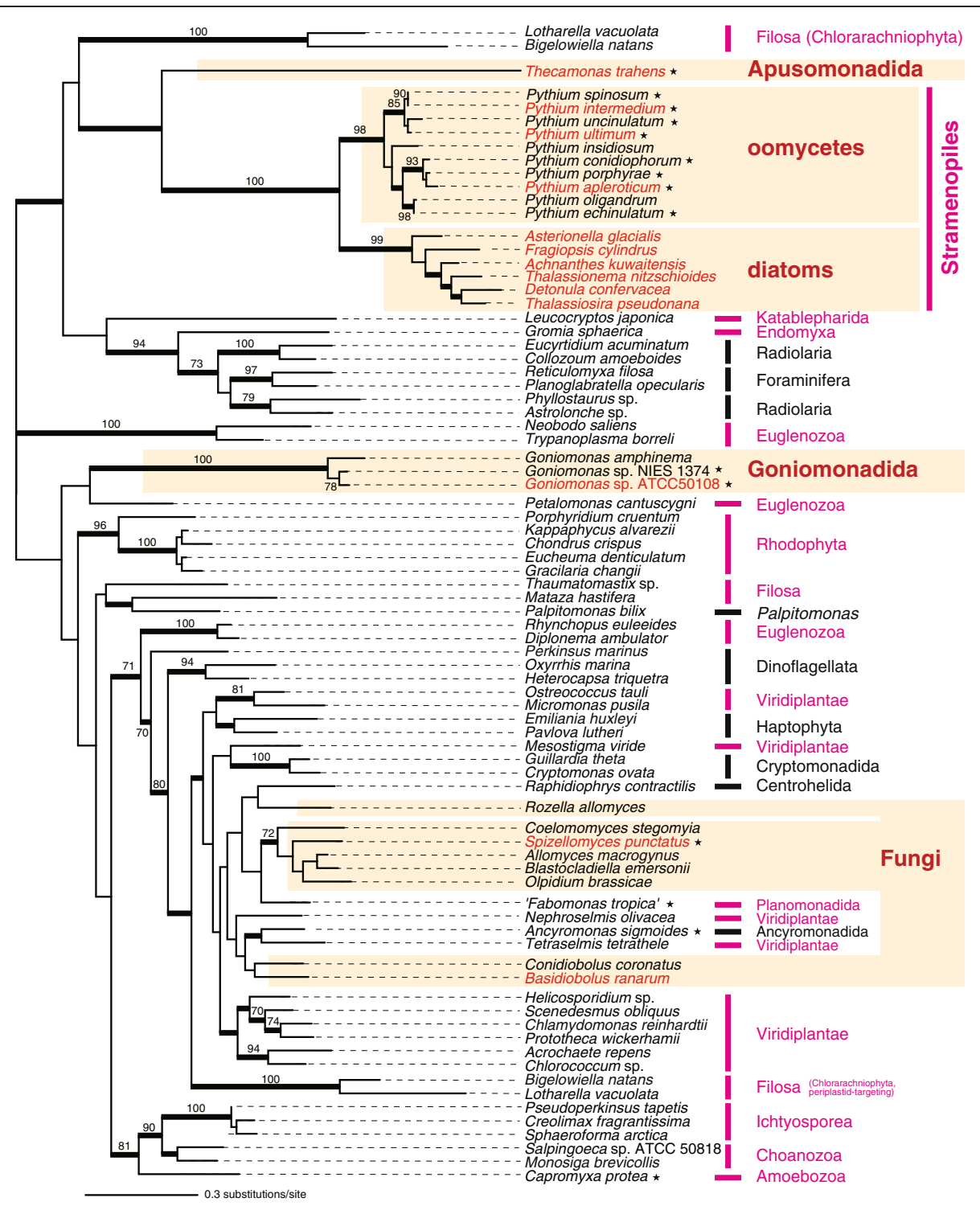

Figure 2 EFL phylogeny. The unrooted maximum-likelihood tree was inferred from 80 EFL sequences with 407 amino acid positions. Only bootstrap values $\geq 70 \%$ are shown. The nodes supported by Bayesian posterior probabilities $\geq 0.95$ are highlighted by thick lines. All other details of the figure are as described in the legend to Figure 1.

(Table 3). In sharp contrast, no contig for EF-1 $\alpha$ was obtained in the transcriptomic data (Table 3), even though our RT-PCR successfully detected EF- $1 \alpha$ transcripts in $P y$. ultimum (data not shown).

\section{Dual-EF-containing species in goniomonads}

Prior to this study, EF- $1 \alpha /$ EFL data were available for only two goniomonad species: EF- $1 \alpha$ transcripts were detected in Goniomonas pacifica [17], while an EFL gene was isolated from Goniomonas amphinema [18]. In this study, we experimentally surveyed EF- $1 \alpha / E F L$ sequences in five Goniomonas strains (ATCC 50108, ATCC PRA68, NIES-1373, NIES-1374, and CCAP 980_1). Of these, strain ATCC 50108 appeared to be dual-EF-containing (Table 1). A qRT-PCR assay revealed that EFL transcripts were more abundant than EF- $1 \alpha$ transcripts in strain ATCC 50108 (Table 2).

The EF-1 $\alpha$ sequences amplified from strains ATCC 50108, ATCC PRA68, NIES-1373, and CCAP 980_1, together with that of G. pacifica, formed a clade in the EF- $1 \alpha$ phylogeny $(\mathrm{MLBP}=58 \%$; node D in Figure 1$)$. The new EFL homologues from strains NIES 1374 and ATCC 50108 showed a close relationship to the G. amphinema homologue (Figure 2). Both EF- $1 \alpha$ and EFL phylogenies suggest vertical inheritance of the genes encoding the two elongation factors in this lineage. 
Table 2 Relative copy numbers of EF-1a and EFL transcripts by qRT PCR

\begin{tabular}{lllll}
\hline Organism & EFL & (sd) & EF-1a & (sd) \\
\hline Goniomonas sp. ATCC 50108 & 1.50 & $(0.28)$ & $4.10 \times 10^{-4}$ & $\left(7.63 \times 10^{-5}\right)$ \\
Asterionella glacialis & 38.77 & $(6.29)$ & 0.09 & $(0.04)$ \\
Achnanthes kuwaitensis & 23.22 & $(1.52)$ & 0.01 & $(0.002)$ \\
Detonula confervacea & 0.08 & $(0.03)$ & $4.88 \times 10^{-4}$ & $\left(0.11 \times 10^{-4}\right)$ \\
Thalassionema nitzschiodes & 0.33 & $(0.10)$ & $6.36 \times 10^{-4}$ & $\left(9.55 \times 10^{-4}\right)$ \\
\hline
\end{tabular}

Notes-normalized by the copy number of a-tubulin transcripts.

\section{Other dual-EF-containing species in Apusomonadida and Fungi}

We detected both EFL and EF-1 $\alpha$ sequences in both whole-genome shotgun and transcriptomic data from the apusomonad Thecamonas trahens (http://www. broadinstitute.org/). The EF-1 $\alpha$ sequences of two apusomonads, T. trahens and Apusomonas proboscidea, grouped together in the $\mathrm{ML}$ tree topology $(\mathrm{MLBP}=$ 37\%; node E in Figure 1), consistent with their organismal relationship. The large discrepancy in branch length between the two apusomonad sequences is likely responsible for the low ML bootstrap support. In the EFL phylogeny, the $T$. trahens sequence branched at the base of the diatom-oomycete clade (Figure 2). Unfortunately, the current analysis does not allow us to determine if EFL genes were the result of descent through vertical inheritance in apusomonads, because: (i) only one EFL sequence is known for apusomonads, and (ii) T. trahens and opisthokonts were distant from each other in the EFL phylogeny, in contrast to the close organismal relationship between apusomonads and opisthokonts e.g., [19].

Our EF-1 $\alpha /$ EFL gene survey also identified the genome of the chytridiomycote fungus Spizellomyces punctatus as encoding both kinds of elongation factors. The EF- $1 \alpha$ sequences of S. punctatus and B. ranarum bore the wellknown Opisthokonta-specific insertion (Additional file 1), and formed a clade with other fungal sequences in the phylogenetic analyses (MLBP $=41 \%$; node F in Figure 1$)$, suggesting that the EF-1 $\alpha$ genes of $S$. punctatus and $B$. ranarum and those of other fungal species share an exclusive ancestry. Again, the grouping of the two longbranched sequences of $S$. punctatus and B. ranarum with other fungal sequences did not receive high ML bootstrap support. We are currently unsure whether the extant EFL

Table 3 k-mer frequencies for EF-1a, EFL, and a-tubulin in transcriptomic data

\begin{tabular}{lllll}
\hline & & \multicolumn{3}{l}{ k-mer frequency } \\
\cline { 3 - 5 } Organisms & Data sources & EFL & EF-1a & a-tubulin \\
\hline Thecamonas trahens & SRR343042 & 1540 & 21 & 530 \\
Spizellomyces punctatus & SRR343043 & 4797 & 7 & 805 \\
Pythium ultimum & SRR059026 & 556 & Not detected & 31 \\
\hline
\end{tabular}

genes in fungi are the descendents of a single gene in the ancestral fungal species: The monophyly of fungi was not recovered in the ML tree inferred from the EFL alignment (Figure 2), but the approximately unbiased test [20] failed to reject the alternative hypothesis, in which all fungal EFL sequences were enforced to be monophyletic, at the $5 \%$ level (data not shown).

In both $T$. trahens and $S$. punctatus there is a large difference in transcriptional levels between EF-1 $\alpha$ and EFL genes. In the transcriptomic data of the two species, the k-mer frequency for EFL was much greater than that for EF-1 $\alpha$ (Table 3), as seen in other dual-EF-containing species (see above).

\section{New EF-1a/EFL data from other eukaryotes}

Our EF-1 $\alpha /$ EFL survey successfully revealed that the taxa Katablepharida, Amoebozoa (or a subgroup of Amoebozoa), and Ancyromonadida contain both EFLcontaining and EF- $1 \alpha$-containing species. For amoebozoans and ancyromonads, only EF-1 $\alpha$-containing species were known prior to this study (see Figure 1), however, we detected EFL sequences in the amoebozoan Copromyxa protea and the ancyromonad Fabomonas tropica (Table 1). Likewise, the first-surveyed katablepharid Leucocryptos marina was EFL-containing [16], but a RT-PCR survey of a secondly-surveyed katablepharid, Roombia sp., identified EF-1 $\alpha$ transcripts (Table 1 ).

\section{Discussion}

Several eukaryote lineages include multiple dual-EFcontaining species

Ancestral co-occurrence of EF- $1 \alpha$ and EFL followed by differential loss of one of the two elongation factors most likely shaped the current EF-1 $\alpha / E F L$ distribution within eukaryotes. In this scenario, the extant dual-EF-containing species retain the ancestral state and thus are analogous to the inferred intermediates that led to descendant lineages that contain either EF-1 $\alpha$ or EFL (Figure 3). In this study, we found 11 new dual-EF-containing species in four distantly related lineages: (1) Goniomonadida, (2) Apusomonadida, (3) Stramenopiles (including diatoms and oomycetes), and (4) Fungi (including S. punctatus and $B$. ranarum). In light of the differential loss process proposed for EF-1 $\alpha /$ EFL evolution, we speculate that more dual-EF-containing species remain undetected in other lineages that contain both EF- $1 \alpha$-containing and EFL-containing species, including: Viridiplantae [6], Euglenozoa [8], Choanoflagellata [5], Endomyxa [10], Filosa [9], Rhodophyta [18], Katablepharida (this study), Amoebozoa (this study), and Ancyromonadida (this study) (highlighted in pink in Figures 1 and 2). Considering the revised distribution of EF-1 $\alpha /$ EFL genes, we cannot exclude the possibility that the last eukaryotic common ancestor was dual-EF-containing. 


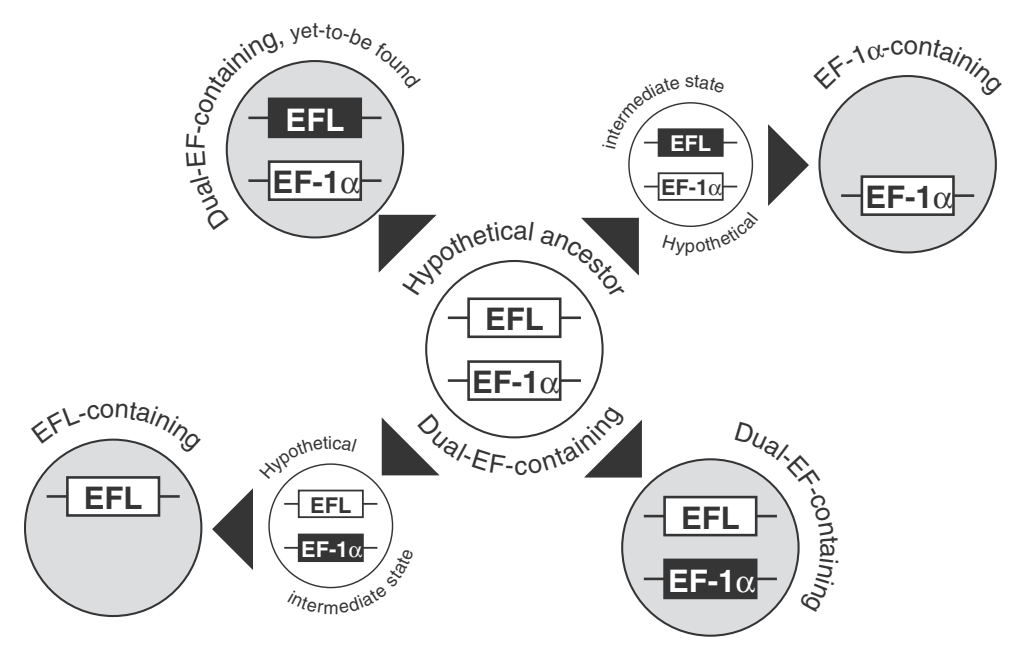

Figure 3 Scheme for EF-1a/EFL evolution in eukaryotes. A differential loss process from the hypothetical dual-EF-containing ancestor (center; open) produced four descendent types (shaded): (i) EFL-containing descendent (lower left), (ii) EF-1a-containing descendent (upper right), (iii) dual-EF-containing descendent with a transcriptionally suppressed EF-1a (lower right), and (iv) dual-EF-containing descendent with a transcriptionally suppressed EFL gene (upper left). The EF-1a gene is blackened in the descendent shown in lower right, as this gene is functionally reduced and transcriptionally suppressed, which is likely analogous to the hypothetical intermediate that leads to the EFL-containing type that lacks EF-1a. Likewise, the other type of dual-EF-containing descendent (upper left), if exist, bears the re-modeled EFL gene (blackened), and is analogous to the hypothetical intermediate that led to the EF-1a-containing descendants that lack EFL.

Finally, it will be of interest to continue surveying dualEF-containing species, especially within Stramenopiles and Fungi. Kamikawa et al. [16] postulated that the dual-EF status can be traced back to the ancestral stramenopile species, based on the monophyly of stramenopiles in EF-1 $\alpha$ phylogenies (Figure 1), and of diatoms and oomycetes in EFL phylogenies (Figure 2: Note that no EFL homologue has been identified to date in any stramenopile subgroups except diatoms and oomycetes). Thus, we predict that dual-EF-containing species should be found in so-far unsampled stramenopiles. Similarly, S. punctatus and B. ranarum are unlikely to be the sole fungal species with a dual-EF status, given that the most recent common ancestral fungus was proposed to be dual-EF-containing [12].

\section{Parallel re-modeling of EF-1a function in eukaryotic evolution}

In the dual EF-containing diatom T. pseudonana, some of us [7] proposed that the EF- $1 \alpha$ homolog performs only a subset of its original functions, and does not participate in protein synthesis as an elongation factor, for the following reasons. Firstly, in an EF-1 $\alpha$ phylogeny, the T. pseudonana homologue was much more divergent than that of a closely related EF- $1 \alpha$-containing species, $P$. tricornutum, suggesting that the former is under fewer functional constraints than the latter. Secondly, EF-1 $\alpha$ transcripts were much less abundant in T. pseudonana than the transcripts of EFL or of an $\alpha$-tubulin gene. As observed in $T$. pseudonana, the five dual EF-containing diatoms identified in this study (i.e. A. kuwaitensis, A. glacialis, D. confervacea, F. cylindrus, and T. nitzschioides) appeared to possess divergent EF-1 $\alpha$ genes (Figure 1). In each of the five diatoms, the transcriptional level of the EF-1 $\alpha$ gene was heavily suppressed compared to that of the co-occurring EFL gene (Table 2). Thus, the five dualEF-containing diatoms most likely use EFL as the principal elongation factor, while a sub-set of the original EF- $1 \alpha$ functions is assigned to the divergent EF-1 $\alpha$. These dualEF-containing diatoms have most likely re-modeled their EF-1 $\alpha$ functions, such that they carry out only the auxiliary roles that the proteins originally performed, such as interactions with cytoskeletal proteins and ubiquitindependent protein degradation $[1,21,22]$.

It is likely that similar re-modeling of EF- $1 \alpha$ function has also occurred in other dual-EF-containing lineages. In the non-diatom dual-EF-containing species, the EF$1 \alpha$ sequences were also divergent (Figure 1), and were transcribed at a low level compared to the co-occurring EFL genes (Tables 2 and 3). These results strongly suggest that dual-EF-containing species in general utilize EF- $1 \alpha$ for subsets of the original functions, while EFL participates in translation as a core factor. Significantly, the re-modeling of EF-1 $\alpha$ function probably took place separately in Stramenopiles (including diatoms and oomycetes), Goniomonadida, Apusomonadida, and Fungi, as these lineages are distantly related to one another in the organismal phylogeny. Moreover, diatoms (photosynthetic heterokont algae) and oomycetes (non-photosynthetic stramenopiles) may have also re-modeled their EF-1 $\alpha$ functions in parallel as they are relatively distantly related within stramenopiles. We also suspect 
that parallel re-modeling of EF- $1 \alpha$ function occurred within Fungi, as $S$. punctatus and B. ranarum are not particularly close relatives [12].

We are currently unsure about the precise functions of the divergent EF- $1 \alpha$ in the dual-EF-containing species. Under the parallel re-modeling scenario proposed above, the suite of retained EF- $1 \alpha$ functions could vary between any of two dual-EF-containing lineages. However, the overall substitution patterns in divergent EF-1 $\alpha$ sequences in distantly related dual-EF-containing species are found to be similar to each other (Additional file 2). This observation hints at parallel loss of the same aspects of EF-1 $\alpha$ function and retention of a subset of original functions in multiple dual-EF-containing lineages scattered over the tree of eukaryotes. These speculations could be tested more directly by biochemical studies of EF-1 $\alpha$ function in selected representatives of these lineages.

\section{Conclusions}

According to the differential loss hypothesis for EF-1 $\alpha$ / EFL evolution, a dual-EF-containing ancestor likely gave rise to two types of descendants-one containing only EFL and the other containing only EF-1 $\alpha$. Nevertheless, EF-1 $\alpha /$ EFL surveys, including this study, have identified an additional type of descendent retaining the ancestral arrangement (i.e. dual-EF-containing) in multiple branches of the tree of eukaryotes. If EF-1 $\alpha / E F L$ sequences are surveyed in a broader spectrum of eukaryotes, it is highly likely that the number and diversity of known dual-EFcontaining species will grow further.

Curiously, all dual-EF-containing species identified so far appear to retain divergent, low-expressed EF-1 $\alpha$ genes (see above), which are analogous to the hypothetical intermediate leading to EFL-containing descendants (Figure 3). We suspect that the multiple functions of the canonical EF-1 $\alpha$ may have prevented the dual-EFcontaining cells from losing this protein immediately after EFL took over from EF- $1 \alpha$ as the core translation factor. The presence of dual-EF-containing species indicates that the adoption of EFL as the dominant core factor in translation does not necessarily lead to the elimination of EF- $1 \alpha$ from the entire cellular system.

Curiously, we found little evidence for living analogues of the hypothetical intermediate that led to EF-1 $\alpha$ containing descendants, which would possess a divergent, low-transcribed EFL gene. The presence or absence of dual-EF-containing species, in which a divergent EFL gene is transcribed at lower levels than the co-occurring EF-1 $\alpha$ gene, would be crucial to understanding the evolutionary processes that shaped the current EF-1 $\alpha / E F L$ gene distribution across the tree of eukaryotes. We need to re-examine EFL sequences in the species currently recognized as 'EF-1 $\alpha$-containing' since low-expressed
EFL genes might be overlooked in these taxa, especially if genomic or high-coverage transcriptomic data is lacking.

\section{Methods}

Strains

Achnanthes kuwaitensis (NIES-1349), Asterionella glacialis (NIES-417), Thalassionema nitzschioides (NIES-534), Goniomonas amphinema (NIES1371), Goniomonas truncata (NIES-1373), and Goniomonas sp. (NIES-1374) were purchased from the Microbial Culture Collection at the National Institute for Environmental Study in Japan. Detonula confervacea (CCMP353) and Bolidomonas pacifica (CCMP1866) were purchased from the ProvasoliGuillard National Center for Marine Algae and Microbiota. Goniomonas sp. (CCAP 980/1) was purchased from the Culture Collection of Algae and Protozoa. Goniomonas sp. (ATCC PRA-68) and Goniomonas sp. (ATCC 50108) were purchased from American Type Culture Collection. Pythium apleroticum (MAFF425515), Py. conidiophorum (MAFF245320), Py. echinulatum (MAFF425510), Py. intermedium (MAFF306022), Py. porphyrae (MAFF239483), Py. spinosum (MAFF425453), Py. ultimum (MAFF425505), and Py. uncinulatum (MAFF240295) were purchased from the GeneBank (Microorganism Section) at the National Institute of Agrobiological Sciences in Japan. Roombia sp. strain NY0200 was cultivated with bacterial prey in URO-YT medium (Moriya et al. 2000). RNA was extracted from the harvested cells by using an RNeasy Plant Mini kit (QIAGEN), and then subjected to oligo (dT)-primed reverse transcriptase (RT) reactions by using the 3' rapid amplification of cDNA ends kit (Invitrogen). Each of the two procedures described above was conducted following the corresponding manufacturers' instructions.

\section{PCR-based survey of EF-1a and EFL transcripts}

We amplified EF-1 $\alpha$ and/or EFL sequences of Roombia sp., diatoms, Bolidomonas pacifica, and goniomonads (see the previous section) by a two-step procedure: For the first RT-PCR, the combination of one of three forward primers (5'-GGCCACGTGGAYTCNGGNAARTCNAC, 5' -GGC CACGTGGAYAGYGGNAARTCNAC, or 5'-GGCCACG TGGAYGCNGGNAARTCNAC) and a reverse primer (5'-ACGAAATCTCTCTTRTGNCCNGGNGCRTC) were used. These primer sets can amplify the 5 ' portions of the transcripts ( $\sim 250 \mathrm{bp}$ in length) for EF-1 $\alpha$ and EFL, as well as other EF-1 $\alpha$-related proteins in a single reaction. For each reaction, amplicons were cloned into pGEMTEasy vector (Promega), and sequenced $\geq 12$ clones to survey EF-1 $\alpha /$ EFL sequences. Secondly, the 3 ' portions of Roombia, diatom, and goniomonad EF-1 $\alpha /$ EFL transcripts were amplified by the 3 ' rapid amplification of cDNA ends (RACE) kit (Invitrogen) with exact-match primers based on the nucleotide sequences of the initial 
amplicons. We amplified the 3' portion of the EF-1 $\alpha$ transcript of $B$. pacifica by the combination of an exactmatch primer (see above) and a degenerate primer, which can anneal to the 3 ' portion of EF- $1 \alpha$ open reading frame (5'-CAGAATTGCGACAGCNACNGTYTG). Amplicons were cloned and sequenced completely as described above.

From all of the seven species belonging to the oomycete genus Pythium examined in this study, we obtained the amplicons covering most of the EFL-coding region by RTPCR with a set of primers 5 '-AGCCGAGAAGGGTGG TTTCG and 5'-ACAGATAATCTGACCAACACC. The details of cloning and sequencing of the EFL amplicons were same as described above.

We then screened the $5^{\prime}$ portion of EF-1 $\alpha$ sequences in the seven Pythium spp. in two separate trials. Firstly, we applied the combinations of primers for EF-1 $\alpha$ sequences in phylogenetically diverse eukaryotes; two forward primers (5'-GTGGACGCCGGNAARTCNACN ACNAC and $5^{\prime}$-GTGGACGCCGGNAARAGYACNAC NAC) and two reverse primers (5' ${ }^{\prime}$ TCGGCCTGGGAN GTNCCNGTNATCAT and 5'-TCGGCCTGGGTNGT NCCNGTNATCAT). The RT-PCR with these 'universal' primers succeeded in amplifying the partial EF- $1 \alpha$ transcripts in Py. apleroticum. For the second trial, we prepared new degenerate primers, which were more specific to oomycete EF- $1 \alpha$ sequences than those used in the first trial: PytEF1aFA, PytEF1aFB, and PytEF1aR (5' -TCGGC AAGACGTCGTWCAAGTAC, 5'-GGTCACCGCGATT TCATCAAGAAC, and 5'-GACNGGNACCGTGCCAA TACC, respectively). EF- $1 \alpha$ transcripts in the Pythium spp. were surveyed by the hemi-nested RT-PCR, in which the combination of PytEF1aFA and PytEF1aR, and that of PytEF1aFB and PytEF1aR were used for the first and second reactions, respectively. The partial EF- $1 \alpha$ transcript in Py. intermedium was amplified in the second trial with the 'oomycete-oriented' primers. We could not detect any EF- $1 \alpha$ transcripts in the Pythium species examined in this study, other than Py. apleroticum and Py. intermedium. The 3' portions of Py. apleroticum and Py. intermedium EF- $1 \alpha$ transcripts were amplified by the $3^{\prime}$ RACE, followed by cloning and sequencing. The details of the 3 ' $\mathrm{RACE}$, and cloning and sequencing of the amplicons were same as described above.

\section{Illumina transcriptomic analyses}

We obtained transcriptomic data from the following organisms; two ancyromonads, Ancyromonas sigmides B70 (CCAP 1958/3) and Fabomonas tropica NYK3C, the breviates, Breviata-like biflagellate PCbi66 and Subulatomonas sp. PCMinv5, the mantamonad Mantamonas plastica Bass1 (CCAP 1946/1), the tubulinid amoebozoan Capromyxa protea CF08-5 (ATCC PRA$324)$, and the microaerophilic cercozoan strain DMV.
A. sigmoides and 'F. tropica' were cultivated with bacterial prey (Enterobacter aerogenes) in a mixture of $50 \%$ ATCC 802 medium and 50\% filtered sterile seawater, and in a mixture of $50 \%$ seawater and $50 \% \mathrm{ddH}_{2} \mathrm{O}$, respectively. Strain PCbi66 was grown in ATCC 1525 medium with bacterial prey (Klebsiella pneumoniae ATCC 23432). Subulatomonas sp. was cultivated with bacterial prey in ATCC 1773 medium made with 50\% seawater and $50 \% \mathrm{ddH}_{2} \mathrm{O}$. M. plastica was grown with bacterial prey (K. pneumoniae ATCC 23432) in a mixture of 50\% seawater and $50 \% \mathrm{dd}_{2} \mathrm{O}$. C. protea was grown on weak malt yeast agar plates $(0.02 \mathrm{~g}$ Yeast extract, $0.02 \mathrm{~g}$ Malt extract, $0.75 \mathrm{~g} \mathrm{~K}_{2} \mathrm{HPO}_{4}, 1 \mathrm{~L} \mathrm{ddH}_{2} \mathrm{O}, 15 \mathrm{~g}$ Agar) with streaks of Escherichia coli as food. Stain DMV was grown in ATCC 802 medium, with bacterial prey (K. pneumoniae ATCC 23432) killed at $65^{\circ} \mathrm{C}$ for 1 hour.

Total RNA was isolated using Trizol (Tri-reagent) following the protocol supplied by the manufacturer (Sigma). Construction of cDNA libraries and illumina RNAseq was performed by Macrogen (South Korea) for strain PCbi66 and A. sigmoides, by GeneWiz (USA) for 'F. tropica', Subulatomonas sp., and M. plastica, and by the Institut de Recherche en Immunologie et Cancérologie (IRIC) of Universite de Montreal (Canada) for C. protea and strain DMV.

Raw sequence read data were filtered based on quality scores with the fastq_quality_filter program of FASTXTOOLS (http://hannonlab.cshl.edu/fastx_toolkit/), using a cutoff filter (a minimum $70 \%$ of bases must have quality of 20 or greater). Filtered sequences were then assembled into clusters using the Inchworm assembler of the TRINITY r2001-5-13 package [23]. EF-1 $\alpha /$ EFL sequences were identified using basic local alignment search tool (tblastn).

\section{Database search of EFL and EF-1a genes}

By using $T$. pseudonana EFL and EF- $1 \alpha$ amino acid sequences as the queries, we performed tblastn searches with $E$-value cutoff $<10^{-100}$. Putative EF-1 $\alpha / E F L$ sequences identified by the initial tblastn search were then confirmed by blastp searches with $E$-value cutoff $<10^{-100}$. The reciprocal similarity searches identified both EFL and EF-1 $\alpha$ genes in the genomes of T. trahens and S. punctatus from the whole genome shotgun database in NCBI (http://www.ncbi.nlm.nih.gov/). Likewise, both EF-1 $\alpha$ and EFL genes were detected in the genome databases of the diatom F. cylindrus (http://genome.jgi-psf.org/ Fracy1/Fracy1.home.html) and the oomycete Py. ultimum (http://pythium.plantbiology.msu.edu/). For the Illumina RNAseq data of T. trahens, S. punctatus, and Py. ultimum we collected raw sequence data from the NCBI's Short Reads Archive (SRA), accessions SRR343042, SRR343043, and SRR059026, respectively. These raw data were assembled into clusters using the Inchworm assembler of 
the TRINITY r2001-5-13 package, as above. We then identified the contigs pertaining to EFL, EF- $1 \alpha$, and $\alpha$ tubulin through tblastn, and compared the k-mer frequency of each respective contig to compare the relative transcriptional level between the co-occurring EFL and EF-1 $\alpha$ genes (Table 3). We provide the amino acid sequences mentioned here as Additional file 3.

\section{Phylogenetic analysis}

EFL and EF- $1 \alpha$ amino acid sequences were sampled from the broad spectrum of eukaryotes. Datasets of the two elongation factor families were separately aligned, and then ambiguously aligned positions were excluded before phylogenetic analyses. The final EFL and EF- $1 \alpha$ datasets contained 80 sequences with 407 amino acid positions and 79 sequences with 400 amino acid positions, respectively. The two datasets were analyzed using both ML and Bayesian phylogenetic methods. ML analyses were performed using RAxML 7.2.1 [24] under the LG model [25] incorporating empirical amino acid frequencies and among-site rate variation approximated by a discrete gamma distribution with four categories $(\mathrm{LG}+\Gamma+\mathrm{F}$ model). The ML tree was estimated by heuristic searches based on 300 distinct parsimony starting trees. In RAxML bootstrap analyses (1000 replicates), the heuristic tree search was performed from a single parsimony tree per replicate.

The EFL and EF- $1 \alpha$ datasets were also subjected to Bayesian analysis using PhyloBayes v.3.3 [26] with the $\mathrm{LG}+\Gamma+\mathrm{F}$ model. For the EF-1 $\alpha$ analysis, two parallel Markov Chain Monte Carlo (MCMC) runs were run for 63,799 and 63,885 generations, sampling log-likelihoods and every 10 trees (maxdiff $=0.16254$; 'burn-in' was set as 100 based on the log-likelihood plots). The EFL dataset was analyzed as described above, except two MCMC runs were run for 12,520 and 12,511 generations $($ maxdiff $=0.113078)$.

\section{Quantitative reverse transcriptase (qRT) PCR}

To normalize the copy numbers of EFL and EF- $1 \alpha$ transcripts, we amplified the $\alpha$-tubulin sequence of Goniomonas sp. ATCC 50108 by RT-PCR with the following degenerate primers: 5'-RGTNGGNAAYGCNTGY TGGGA and $5^{\prime}$-CCATNCCYTCNCCNACRTACCA. To amplify the $\alpha$-tubulin sequences of diatoms $A$. kuwaitensis, A. glacialis, and T. nitzschioides, we used a second set of degenerate primers: 5'-GARCTNTAYTGYCTNGARCA YGG and 5'-CGCGCCATNCCYTCNCCNACRTACCA. The $\alpha$-tubulin sequence of the diatom $D$. confervacea was amplified by using the following primers: 5 '-CGCGCC ATNCCYTCNCCNACRTACCA and 5'-CGTAGANAG CCTCGTTGTC. The cloning and sequencing of the $\alpha-$ tubulin amplicons were carried out as described above.
Accession nos. for the sequences are AB766056 AB766059.

In Table 4 we list the exact-match primers used for qRT-PCR assays designed based on the EF- $1 \alpha$, EFL, and $\alpha$-tubulin sequences in the four diatoms and Goniomonas sp. ATCC 50108. The plasmids carrying the EFL, EF- $1 \alpha$, and $\alpha$-tubulin amplicons (see above) were used as the standards for qRT-PCR. A mixture for qRT-PCR contained SYBR Green I (TaKaRa), Premix ExTaq (TaKaRa), a set of exact-match primers (final concentration of $0.3 \mu \mathrm{M}$ each), and template solution:

Table 4 Primers and annealing temperatures for qRT PCR

\begin{tabular}{|c|c|c|}
\hline Organisms & Genes $\left({ }^{\circ} \mathrm{C}\right)^{*}$ & Primers \\
\hline \multirow{6}{*}{$\begin{array}{l}\text { Achnenthes } \\
\text { kuwaitensis }\end{array}$} & EFL (57) & 5'-GTCACTTGATCTTCAAGCAG \\
\hline & & 5'-TGTCGGTGAAGAACTCCTTG \\
\hline & $E F-1 a(60)$ & 5'-GAGGAGTTGACGAGAACACG \\
\hline & & 5'-TTGGAGACTCGAACTTCCAG \\
\hline & a-tubulin (60) & 5'-TGGAGCCCTACAACTCCATC \\
\hline & & 5'-CACCAGGTTGGTCTGGAACTC \\
\hline \multirow{6}{*}{$\begin{array}{l}\text { Asterionella } \\
\text { glacialis }\end{array}$} & EFL (58) & 5'-TATCTCTGAGCGTGAGATGAAG \\
\hline & & 5'-CTTGGTGTTGCACTGAATGG \\
\hline & EF-1a (54) & 5'-TGAAGAACGAACTATGGAAG \\
\hline & & 5'-CCAAAGTGAAATATCGATTG \\
\hline & a-tubulin (58) & 5'-ACATGGCATGCTGCCTCATG \\
\hline & & 5'-ATCCTCGAAAGAGCTTCTGC \\
\hline \multirow{6}{*}{$\begin{array}{l}\text { Detonula } \\
\text { confervacea }\end{array}$} & EFL (58) & 5'-AGGAATCTCTGCTCGTGAG \\
\hline & & 5'-GAACTCCTTGGTGTTACACTG \\
\hline & EF-1a (58) & 5'-GAAACCATCGACAAGTACG \\
\hline & & 5'-GAAACTTCCACAACGTGATATCG \\
\hline & a-tubulin (58) & 5'-CAAATGCGCAGCGACAAGAC \\
\hline & & 5'-TTCCAGAACGGACCTCGTC \\
\hline \multirow{6}{*}{$\begin{array}{l}\text { Thalassionema } \\
\text { nitzschioides }\end{array}$} & EFL (56) & 5'-AATCTCTGCTCGAGAGATGG \\
\hline & & 5'-TGTAGTGGTACTTGCCAGTG \\
\hline & $E F-1 a(56)$ & 5'-CGTAGCCGAAAGCATAATAG \\
\hline & & 5'-CCAGACACTGATATCAATAG \\
\hline & a-tubulin (56) & 5'-TTGTATGATGTCTGCCGTGG \\
\hline & & 5'-AAGCCTTCTCACGCGAAATA \\
\hline \multirow[t]{6}{*}{ Goniomonas sp. } & EFL (60) & 5'-CATCAAGGGTCTCAAGAAGGACAAC \\
\hline & & 5'-CAGTTGATGGCGGTCATCTTCATG \\
\hline & $E F-1 a(60)$ & 5'-GTTCTCTGCTGGATACACTCCAGTG \\
\hline & & 5'-ACGCTATTCATGGAAGGCCTCAAC \\
\hline & a-tubulin (55) & 5'-CATGTACCGTGGTGATGTCG \\
\hline & & 5'-CTGGACCTTGGCAAGATCAC \\
\hline
\end{tabular}

${ }^{*}$ Numbers in parentheses show primer set-specific annealing temperatures used in qRT PCR. 
either cDNA, the corresponding RNA sample (the negative control), or five differently diluted plasmid solutions including 10 to $10^{7}$ copies of the target gene fragments (the standards). The qRT-PCR thermal cycling conditions were $95^{\circ} \mathrm{C}$ for $30 \mathrm{sec}$ followed by 50 cycles comprised of $95^{\circ} \mathrm{C}$ for $5 \mathrm{sec}$, a gene-specific temperature for $10 \mathrm{sec}$ (Table 4), and $72^{\circ} \mathrm{C}$ for $10 \mathrm{sec}$. We confirmed that a single target product was amplified by real-time PCR, based on melting curves (data not shown). In each assay, the target amplification from the RNA sample was out of the quantifiable range. Smart Cycler II (Cepheid) and Thermal Cycler Dice (TaKaRa) were used for the assays on the four diatoms and that of Goniomonas sp., respectively.

\section{Accession numbers}

AB766030-AB766059, AB775895, and AB824019.

\section{Additional files}

Additional file 1: Partial alignment of EF-1a sequences. The Opisthokonta-specific insertion is highlighted in grey. Numbers above the alignment are the amino acid positions in Thalassiosira pseudonana EF-1a. The divergent EF-1a homologues in the two dual-EF-containing fungi are highlighted by stars.

Additional file 2: Substitution patterns in the divergent EF-1a sequences. The amino acid sequences of the divergent EF-1a homologues (marked by stars) were compared to those of phylogenetically related, canonical EF-1a proteins. Amino acids are grouped into four Dayhoff categories-(i) acidic residues ( $D$ and $E$ ), (ii) basic residues $(H, K$, and $R$ ), (iii) polar-uncharged residues $(C, N, Q, S, T, W$, and $Y$ ), and (iv) hydrophobic non-polar residues ( $A, F, G, I, L, M, P$, and $V$ ). Substitutions across two out of the four Dayhoff categories between the divergent and canonical EF-1a sequences are highlighted in red.

Additional file 3: EF-1a/EFL sequences identified in publicly available databases. The amino acid sequences of EF-1a/EFL homologues identified in publicly available databases are listed here.

\section{Competing interests}

Non-financial competing interests.

\section{Authors' contributions}

RK, MWB, and YN determined sequences. RK, MWB, and $Y \mid$ performed phylogenetic analyses. NY, YS, AH, and RG provided research materials. RK and $\mathrm{Yl}$ designed the study and wrote the manuscript. MWB, NY, AGBS, AJR, and $\mathrm{TH}$ helped to draft the manuscript. All authors read and approved the final manuscript.

\section{Acknowledgements}

RK was a research fellow supported by the Japan Society for Promotion of Sciences (JSPS; no. 210528). NY was supported by grants from the Tula Foundation (Centre for Microbial Diversity and Evolution at the University of British Columbia) and the Canadian Institute for Advanced Research, Program in Integrated Microbial Biodiversity (CIfAR IMB program). This work was supported in part by grants from JSPS awarded to RK (no. 24870004), YI (no. 21370031, 23117006) and TH (no. 23405013, 23247038), and by CIfAR IMB program support to AGBS. Work carried out in AJR's laboratory was supported by grant MOP-62809 from the Canadian Institutes of Health Research. AJR acknowledges support from the Canada Research Chairs Program and the CIfAR IMB program.

\section{Author details}

${ }^{1}$ Graduate School of Global Environmental Studies, Kyoto University, Kyoto 606-8501, Japan. ${ }^{2}$ Graduate School of Human and Environmental Studies, Kyoto University, Kyoto 606-8501, Japan. ${ }^{3}$ Centre for Comparative Genomics and Evolutionary Bioinformatics, Department of Biochemistry and Molecular Biology, Dalhousie University, Halifax, NS, Canada. ${ }^{4}$ Graduate School of Life and Environmental Sciences, University of Tsukuba, 1-1-1 Tennoudai, Tsukuba, Ibaraki 305-8572, Japan. ${ }^{5}$ Graduate School of Agriculture, Kyoto University, Kitashirakawa Oiwake-cho, Kyoto 606-8502, Japan. ${ }^{6}$ Department of Biology, Dalhousie University, Halifax NS, Canada. 'Department of Botany, University of British Columbia, 6270 University Blvd., Vancouver, BC V6T 1Z4, Canada. ${ }^{8}$ Center for Computational Sciences, University of Tsukuba, 1-1-1 Tennoudai, Tsukuba, Ibaraki 305-8577, Japan.

Received: 26 April 2013 Accepted: 21 June 2013

Published: 26 June 2013

\section{References}

1. Negrutskii BS, El'skaya AV: Eukaryotic translation elongation factor 1a: structure, expression, functions, and possible role in aminoacyl-tRNA channeling. Prog Nucleic Acid Res Mol Biol 1998, 60:47-78.

2. Dreher TW, Uhlenbeck OC, Browning KS: Quantitative assessment of EF-1a. GTP binding to aminoacyl-tRNAs, aminoacyl-viral RNA, and tRNA shows close correspondence to the RNA binding properties of EF-Tu. J Biol Chem 1999, 274:666-672.

3. Iwabe N, Kuma K, Hasegawa M, Osawa S, Miyata T: Evolutionary relationship of archaebacteria, eubacteria, and eukaryotes inferred from phylogenetic trees of duplicated genes. Proc Natl Acad Sci USA 1989, 86:9355-9359

4. Keeling PJ, Inagaki Y: A class of eukaryotic GTPase with a punctate distribution suggesting multiple functional replacements of translation elongation factor 1a. Proc Natl Sci Acad USA 2004, 101:15380-15385.

5. Ruiz-Trillo I, Lane CE, Archibald JM, Roger AJ: Insights into the evolutionary origin and genome architecture of the unicellular opisthokonts Capsaspora owczarzaki and Sphaeroforma arctica. J Eukaryot Microbiol 2006, 53:379-384.

6. Noble GP, Roger MB, Keeling PJ: Complex distribution of EFL and EF-1alpha proteins in the green algal lineage. BMC Evol Biol 2007, 7:82.

7. Kamikawa R, Inagaki Y, Sako Y: Direct phylogenetic evidence for lateral transfer of elongation factor-like gene. Proc Natl Acad Sci USA 2008, 105:6965-6969.

8. Gile GH, Faktorová D, Castlejohn CA, Burger G, Lang BF, Farmer MA, Lukes J, Keeling PJ: Distribution and phylogeny of EFL and EF-1alpha in Euglenozoa suggest ancestral co-occurrence followed by differential loss. PLoS One 2009, 4:e5162.

9. Kamikawa R, Yabuki A, Nakayama T, Ishida K, Hashimoto T, Inagaki Y: Cercozoa comprises both EF-1a-containing and EFL-containing members. Eur J Protistol 2011, 47:24-28.

10. Ishitani Y, Kamikawa R, Yabuki A, Tsuchiya M, Inagaki Y, Takishita K: Evolution of elongation factor-like (EFL) protein in Rhizaria is revised by radiolarian EFL gene sequences. J Eukaryot Microbiol 2012, 59:367-373.

11. Szabová J, Růžiččka P, Verner Z, Hampl V, Lukeš J: Experimental examination of EFL and MATX eukaryotic horizontal gene transfers: Coexistence of mutually exclusive transcripts predates functional rescue. Mol Evol Biol 2011, 28:2371-2378

12. James TY, Kauff F, Schoch $C L$, et al: Reconstructing the early evolution using a six-gene phylogeny. Science 2006, 443:818-822 (67 co-authors).

13. Bowler $\mathrm{C}$, Allen $\mathrm{AE}$, Badger $\mathrm{JH}$, et al: The Phaeodactylum genome reveals the evolutionary history of diatom genomes. Nature 2008, 456:239-244 (74 co-authors).

14. Daugbjerg N, Guillou L: Phylogenetic analyses of Bolidophyceae (Heterokontophyta) using $\mathrm{rbcL}$ gene sequences support their sister group relationship to diatoms. Phycologia 2001, 40:153-161.

15. Haas BJ, Kamoun S, Zody MC, et al: Genome sequence and analysis of the Irish potato famine pathogen Phytophthora infestans. Nature 2009, 461:393-398 (93 co-authors).

16. Kamikawa R, Sakaguchi M, Matsumoto T, Hashimoto T, Inagaki Y: Rooting for the root of elongation factor-like protein phylogeny. Mol Phylogenet Evol 2010, 56:1082-1088.

17. Hackett JD, Yoon HS, Li S, Reyes-Prieto A, Rümmele SE, Bhattacharya D: Phylogenomic analysis supports the monophyly of cryptophytes and haptophytes and the association of Rhizaria with chromalveolates. $\mathrm{Mol}$ Biol Evol 2007, 24:1702-1713.

18. Sakaguchi M, Takishita K, Tsuchiya M, Hashimoto T, Inagaki Y: Tracing back EFL gene evolution in the cryptomonads-haptophytes assemblage: 
Separate origins of EFL genes in haptophytes, photosynthetic cryptomonads, and goniomonads. Gene 2009, 441:126-131.

19. Derelle R, Lang BF: Rooting the eukaryotic tree with mitochondrial and bacterial proteins. Mol Biol Evol 2012, 29:1277-1289.

20. Shimodaira $\mathrm{H}$ : An approximately unbiased test of phylogenetic tree selection. Syst Biol 2002, 51:492-508.

21. Gonen H, Smith CE, Siegel NR, Kahana C, Merrick WC, Chakraburtty K, Schwartz AL, Ciechanover A: Protein synthesis elongation factor EF-1alpha is essential for ubiquitin-dependent degradation of certain $\mathrm{N}$ alphaacetylated proteins and may be substituted for by the bacterial elongation factor EF-Tu. Proc Natl Acad Sci USA 1994, 91:7648-7652

22. Liu G, Edmonds BT, Condeelis J: pH, EF-1a and the cytoskeleton. Trends Cell Biol 1996, 6:168-171

23. Grabherr MB, Haas BJ, Yassour M, et al: (18 co-authors): Full-length transcriptome assembly from RNA-Seq data without a reference genome. Nature Biotechnol 2011, 29:644-652.

24. Stamatakis A: RAxML-VI-HPC: maximum likelihood-based phylogenetic analyses with thousands of taxa and mixed models. Bioinformatics 2006, 22:2688-2690

25. Le SQ, Gascuel O: An improved general amino acid replacement matrix. Mol Biol Evol 2008, 25:1307-1325.

26. Lartillot N, Lepage T, Blanquart S: PHYLOBAYES 3: a Bayesian software package for phylogenetic reconstruction and molecular dating. Bioinformatics 2009, 25:2286-2288.

doi:10.1186/1471-2148-13-131

Cite this article as: Kamikawa et al:: Parallel re-modeling of EF-1a function: divergent EF-1a genes co-occur with EFL genes in diverse distantly related eukaryotes. BMC Evolutionary Biology 2013 13:131.

\section{Submit your next manuscript to BioMed Central and take full advantage of:}

- Convenient online submission

- Thorough peer review

- No space constraints or color figure charges

- Immediate publication on acceptance

- Inclusion in PubMed, CAS, Scopus and Google Scholar

- Research which is freely available for redistribution 